\title{
Acupuncture affects different ways for migraineurs of long- and short- courses on the efficiency of structural brain network
}

\section{Kang Wu}

Beijing University of Chinese Medicine Affiliated Dongzhimen Hospital https://orcid.org/0000-00023684-1552

\section{Kuang Shi Li}

Beijing University of Chinese Medicine Affiliated Dongzhimen Hospital

\section{Ling Ling Xu}

Beijing University of Chinese Medicine Affiliated Dongzhimen Hospital

\section{Pei Chen}

Beijing Anding Hospital Affiliated to Capital Medical University

\section{Lan Jiang}

Beijing University of Chinese Medicine Affiliated Dongzhimen Hospital

\section{Ya Hui Wang}

Beijing University of Chinese Medicine Affiliated Dongzhimen Hospital

\section{Chen Chen}

Beijing University of Chinese Medicine Affiliated Dongzhimen Hospital

\section{Yue Wang}

Beijing University of Chinese Medicine Affiliated Dongzhimen Hospital Hua Lei Geng

Beijing University of Chinese Medicine Affiliated Dongzhimen Hospital

Rui Jia Liu

Beijing University of Chinese Medicine Affiliated Dongzhimen Hospital

\section{Yi Ren}

Beijing University of Chinese Medicine Affiliated Dongzhimen Hospital Yi Huai Zou ( $\square$ zouyihuai2004@163.com )

Dongzhimen Hospital affiliated to Beijing University of Chinese Medicine

\section{Research article}

Keywords: Migraine without aura, acupuncture, mechanism, course, structure, brain network, fMRI, graph theory, central sensitization 
Posted Date: July 29th, 2020

DOl: https://doi.org/10.21203/rs.3.rs-48072/v1

License: (c) (i) This work is licensed under a Creative Commons Attribution 4.0 International License. Read Full License 
Acupuncture affects different ways for migraineurs of long- and shortcourses on the efficiency of structural brain network

Kang $\mathrm{Wu}^{1}$, Kuang-Shi $\mathrm{Li}^{1 \dagger}$, Ling-Ling $\mathrm{Xu}^{1}$, Pei Chen ${ }^{2}$, Lan Jiang ${ }^{1}$, Ya-Hui Wang ${ }^{1}$, Chen Chen ${ }^{1}$, Yue Wang ${ }^{1}$, Hua-Lei Geng ${ }^{1}$, Rui-Jia Liu ${ }^{1}$, Yi Ren ${ }^{1 * \dagger}$, Yi-Huai Zou $^{1 *+}$

\section{ABSTRACT}

Background: To study the impact of acupuncture treatment on structural brain network for patients with different courses of migraine without aura based on fMRI.

Methods: A total of ten patients with a long course ( $>5$ years) of migraine without aura (MWoA), ten patients with a short course ( $<5$ years) of MWoA, and ten healthy volunteers were recruited in this trail. All patients received acupuncture treatment three times per week for four weeks. The changes in the topology properties of structural brain regions were observed via fMRI. The clinical symptom scores were adopted for evaluating the therapeutic effect.

Results: 1) The small-world property on the group with a long course was decreased significantly $(p<0.01)$ after treatment, while the group with a short course increased $(p<0.01) .2)$ The clustering coefficient or nodal local efficiency of brain nodes (including the pontine crossing tract, left corticospinal tract, right medial lemniscus, left posterior limb of the internal capsule, right cingulum

\footnotetext{
${ }^{1}$ Dongzhimen Hospital affiliated to Beijing University of Chinese Medicine, Beijing, China

${ }^{2}$ Beijing Anding Hospital, Capital Medical University, Beijing, China

* Corresponding author: Yi-Huai Zou, Email: zouyihuai2004@163.com; Yi Ren, Email: rywendy1982@sina.com.

${ }^{\dagger}$ Kuang-Shi Li, Yi-Huai Zou and Yi Ren contributed equally to this work.
} 
hippocampus, left fornix, left superior longitudinal fasciculus and right tapetum) showed significant changes $(p<0.001)$ with an opposite tendency on the two groups after treatment. 3) The left posterior limb of the internal capsule $(p<0.001, R=-0.73)$ and right tapetum $(p<0.05, R=-0.54)$ had a strong relationship with migraine courses.

Conclusions: The two unequal ways of acupuncture on the structural brain network of migraineurs with different disease courses were revealed. Acupuncture could regulate the central sensitization of chronic migraines, differently with the early stage. The nodal efficiency of the left posterior limb of the internal capsule and right tapetum would be symbols to discriminate against the process of MWoA.

Keywords: Migraine without aura, acupuncture, mechanism, course, structure, brain network, fMRI, graph theory, central sensitization.

\section{Background}

Migraine, a kind of paroxysmal brain dysfunction disease with multi-stage effects of headache and may incorporate neurological symptoms [1], is ranked the second most disabling neurological disorder as well as the third most prevalent medical condition in the world by WHO [2]. In recent years, it was demonstrated that acupuncture could improve the symptoms of migraineurs in the aspects of headache frequency [3], duration, degree as well as attacked days [4]. Several systematic reviews [5-7] corroborated that acupuncture had at least a similar effect as the standard prophylactic drugs, for instance, the flunarizine [8] and propranolol [9], but with fewer side effects and more costeffective [7]. Nevertheless, the functionary mechanism of acupuncture hadn't 
been figured out.

The field of functional neuroimaging provided several conclusions. An electroencephalogram (EEG) study [10] demonstrated that acupuncture improved the efficiency of functional brain networks in delta and alpha bands and promoted the long-range connections of bilateral hemispheres on the healthy individuals. A transcranial magnetic stimulation (TMS) trial [11] suggested that single acupoint stimulation increased the motor cortical excitation and decreased its inhabitation for the healthy volunteers. And a functional magnetic resonance imaging (fMRI) study [12] indicated the modulation effects of acupuncture on the abnormal brainstem activity for migraines. However, fMRI was got widely used since the resting brain networks were proposed [13], especially combing $\mathrm{fMRI}$ and resting brain networks to explore the special performance characteristics of migraineurs. Previous studies detected the functional connectivity of the brain network of migraines and found dramatic changes in the default mode network [14], attentional network [15] and salience network [16]. Two trials [17,18] further found that acupuncture improved the functional connectivity of the right frontoparietal network of migraineurs to moderate the headache. Other researchers [19-21] studied the structural connectivity of the brain network and found the abnormal integration within it. However, seldom study discussed the effect of acupuncture for structural connection of brain network.

Recently years, whether the white matter of migraineurs destroyed was still in dispute. Two population-based imaging studies $[22,23]$ indicated the damages on white matter for migraineurs compared with the healthy controls. Two trials $[24,25]$ also showed the microstructural alterations of the white matter in 
migraineurs. However, several studies [26-28] suggested that migraineurs hadn't significant changes in their white matter. Besides, a case-control study [29] indicated that microstructural white matter changes were not observed in the chronic and episodic migraineurs, yet recent research [30] reported the structural differences in brain white matter between the two of them. It seemed that the white matter of migraineurs was destroyed slowly in the process of migraines [31,32]. Hence, we considered that assigning two different courses of migraines could be better to recognize how acupuncture affected the structural connection of the brain network for migraineurs.

Graph theoretical analysis plays an important role in realizing brain connectivity and could find effective connectivity patterns within complex brain structures [33]. It takes the whole brain as a combined of multiple-interrelated networks [34], which deemed each brain region as a node and only concentrated on the function of the regions rather than the size and location [35]. The combination of graph theory and fMRI could simpler to interpret the study conclusion due to the existing anatomic structure.

Therefore, this research studied how acupuncture affected migraineurs with different courses and aimed to investigate as follows: 1) The effect of acupuncture on structural connectivity of brain network between the two different courses of migraines. 2) The corresponding effective brain regions of this effect.

\section{Methods}

\section{Participants}

This trial consisted of ten patients with a long course ( $>5$ years) of migraine 
(Long course group, LC), ten patients with a short course ( $\leq 5$ years) of migraine (Short course group, SC) and ten healthy controls (Healthy control group, $\mathrm{HC}$ ). The study enrolled subjects at the Dongzhimen Hospital of Beijing University of Chinese Medicine, Beijing, China, from Jan.2018 to Sep.2019, and was approved by the institutional review board at the Dongzhimen Hospital, Beijing University of Chinese Medicine.

Patient groups: Inclusion criteria were as follows: 1) Fitting to the standard of migraine without aura in the international classification of headache disease (ICHD-2) issued by the international headache society in 2004 [36]. 2) Age from 18 to 55 , male or female, with right-handedness. 3) Having no metal implant in the body and no contraindications to MRI testing. 4) Having no depression or other serious mental illness. 5) Having no psychoactive drugs taken in the past 3 months, or vasoactive drugs in the past 2 weeks. 6) Having no habit of taking analgesics for a long time. And exclusion criteria were followed: 1) Having the first headache attack of migraine after 55 years old. 2) Having a history of head injury, cluster headache, or other unclassified headaches. 3) Taking medicine to prevent migraines in recent 4 weeks. 4) Having the serious asymmetry or definite pathological changes in the anatomical structure of the head through the MRI scan. 5) Cannot hold on the times of the MRI scan.

Healthy controls: Included criteria were as follows: 1) Having the matched age and gender to patient groups, with right-handedness. 2) Having no organic and significant functional diseases in the body. 3) Having no contraindications to MRI testing. And exclusion criteria were as follows: 1) Being the menstrual female, pregnant female and lactating female. 2) Having the serious asymmetry or definite pathological changes in the anatomical structure of the head through 
the MRI scan. 3) Having a family history of migraine or other serious mental diseases.

\section{Interventions and study design}

Patient groups received treatment three times per week for four weeks. The types of acupuncture included SJ23 (Si Zhu Kong, unilateral), GB8 (Shuai Gu, unilateral), EX-HN5 (Tai Yang, unilateral), GB20 (Feng Chi, unilateral), LI4 (He Gu, bilateral), LR3 (Tai Chong, bilateral), GB41 (Zu Lin Qi, bilateral), GB34 (Yang Ling Quan, bilateral) and SJ5 (Wai Guan, bilateral), which all were deemed the most effective therapeutic schedule for migraineurs in the clinical care of Chinese medicine referred to the Clinical practice guide of evidencebased acupuncture: Migraine (version 2014). The acupoints location was performed by acupoint name and location of the national standard of China (GB/T 12346-2006), and they were inserted approximate 10-15mn through manual rotation to sought for deqi sensation and then held for 30 minutes.

After subjects were included, the patient groups were received fMRI scan and clinical symptom evaluation (including headache intensity, headache attack days, headache frequency) both twice, before the first treatment and after the final treatment. The healthy control group got one fMRI scan as the baseline.

\section{Data measurements}

The fMRI machine is a $3.0 \mathrm{~T}$ superconducting magnetic resonance scanner (Siemens, Germany) from Dongzhimen Hospital. T1 weighted fast disturbed phase gradient-echo sequence is used to scan the axial position of the structural image. The scanning parameters are follows: echo time $(\mathrm{TE})=3.4 \mathrm{~ms}$, repetition time $(T R)=2530 \mathrm{~ms}$, field of view $=240 \mathrm{~mm} \times 240 \mathrm{~mm}$, data matrix $=$ 
$512 \times 512$, flip angle $=12^{\circ}$.

After original diffusion tensor imaging (DTI) data collected, FMRIB software library (FSL, version 6.0) [37] was used to pick up brain mask, correct eddy current and adjust head movement to decrease artifacts bias. Then, diffusion tensor imaging toolkit (DTI-TK) [38] was adopted to revise voxel location into the original point $[0,0,0]$, which reduced the space and position of brain structure to minimize comparison errors. Afterward, the white matter structural template of Enhanced Human Brain Atlas [39] was performed as a bootstrap template to normalize the size of the brain. After three times affine registrations and six times deformable aligns, the morphological deviation of individual brain structure was optimized to the greatest extent. Lastly the final data was warped into the standard space of bootstrap template, an accurate white matter fiber bundles of the brain structure of the subject were established.

Graph theoretical network analysis toolbox (GRETNA) [40] was further used to extract average fractional anisotropy (FA) value of brain nodes, and then the johns hopkins university (JHU) brain areas template which was offered by Johns Hopkins University was overlapped the FA value to establish the network metrics of structural brain network. After the linear Pearson correlation coefficient was computed for each brain region, the topological attributions of brain networks were obtained. And all subjects were implemented by those steps (Figure 1).

\section{Outcomes measure}

The primary outcomes were the topological attributions of brain regions. It contained the following indicators: small-world property for estimating the 
efficiency of the global brain network, clustering coefficient and nodal local efficiency for estimating the efficiency of nodal brain network [41]. All of these indicators devoted to the measurements of the structural brain network. The differences of these indicators on LC and SC were detected before the first treatment and after the final treatment, with $\mathrm{HC}$ as the baseline. And the latent connections between these indicators and migraine courses were explored further. Besides, the clinical symptom scores were treated as second outcomes, which included headache intensity (Visual Analogue Scale, VAS), headache days and headache frequency to comment on the efficacy of acupuncture treatment.

\section{Statistical analysis}

R software (version 3.6.2 for windows) was performed for statistical analysis. The Shapiro-Wilk test was taken to verify whether the continuous data fitted in Gaussian distribution, considering $p$-value $>0.05$ (two-tailed). If the data fitted in the Gaussian distribution of each group, an independent t-test or paired t-test was further used for two groups comparison. Otherwise, the Wilcoxon test was employed. The strict Bonferroni method was adopted to correct the p-value. For the three groups comparison, the one-way analysis of variance and Kruskal Wallis test were used depending on whether their variances were equal, and the LSD method was taken for multiple comparisons. Chi-square test was used for categorical data. The correlation analysis was applied by the Spearman method. All data displayed in mean (range).

\section{Results}

\section{Baseline of fundamental information}


The gender and age of LC, SC and HC had a non-significant level ( $p>0.05)$, which showed the fundamental of the three groups were comparable in gender and age (Table 1).

\section{Primary outcomes}

Before the first treatment, LC and SC showed a slightly lower mean value of small-world property than HC. And they were all changed significantly $(p<0.05)$ after the final treatment, with LC decreased and SC increased (Table 2). We further explored the details of the small-world property, found that LC and SC had a higher value in the level 0.05 and 0.10 of network sparsity (solid line in Figure 2) and changed differently (dashed line in Figure 2).

We considered that the $\mathrm{JHU}$ template has forty-eight brain regions, then we adjusted the significant $p$-value of nodal network to 0.001 level (two-tailed). The brain regions that were significantly changed $(p<0.001$, adjusted) between treatment were the left corticospinal tract, left posterior limb of the internal capsule, right cingulum hippocampus, left fornix, left superior longitudinal fasciculus, right tapetum, pontine crossing tract and right medial lemniscus. Among them, the nodal efficiency of the left corticospinal tract, left posterior limb of the internal capsule, left superior longitudinal fasciculus and right tapetum on LC were increased after treatment. While the right cingulum hippocampus, left fornix, pontine crossing tract and right medial lemniscus on LC were decreased. SC showed the opposite (Table 2, Figure 3).

Besides, we detected the relevance between the efficiency of these brain regions and the courses of migraines. The left posterior limb of the internal capsule $(p<0.001, R=-0.734)$ and right tapetum $(p<0.05, R=-0.538)$ both 
showed a negative correlation with the course (Figure 4).

\section{Secondary outcomes}

We found that the headache symptoms (headache intensity, days and frequency) had decreased trend after treatment as expected (Table 1). The headache intensity showed significantly changed $(p<0.05)$, while the headache days and frequency were non-significant $(p>0.05)$.

\section{Discussion}

This research demonstrated that acupuncture reduced the global efficiency of patients with a long course of migraine and improved patients with a short course of migraine. The related brain regions for these changes could be the pontine crossing tract, left corticospinal tract, right medial lemniscus, left posterior limb of the internal capsule, right cingulum hippocampus, left fornix, left superior longitudinal fasciculus and right tapetum. Furthermore, the left posterior limb of the internal capsule and right tapetum were relevant to the courses of migraines.

Previous studies indicated the abnormal global topological attributions, which included high global efficiency, great clustering coefficient and rich-club organization on migraineurs compared with the healthy individuals $[19,20]$. The trial [20] further exhibited a significant association between headache attack duration and the increase of abnormal topological properties. We found the same phenomenon in this study that the two patient groups (LC and SC) had high small-world property than $\mathrm{HC}$ in the level of network sparsity from 0.05 to 0.10 (solid line in Figure 2) before the treatment. We considered that our migraineurs could have formed an integration of abnormal pain-related brain 
circuits, and the pain information was passed to the central regulator though the short path of this integration.

Previous researchers named this abnormal brain circuits as the central sensitization [42,43], which was extensive existed in chronic migraineurs [44]. It was considered that the central sensitization, which included cortical overreaction, brainstem alterations and sensitization of the trigeminal system [45], contributed to the chronic relapse of migraine [46]. According to our results, the rich small-world property on LC could correspond to this abnormal sensitization, and acupuncture reduced the global efficiency after treatment (Table 2). We speculated that acupuncture could moderate the road of central sensitization of patients with a long course of migraines to relieve the headache.

Interestingly, we were surprised to find that acupuncture significantly increased the small-world property on SC (Table 2). This increased global efficiency suggested that acupuncture affected differently on SC and LC. A past trial [10] demonstrated that acupuncture (Zu San Li acupoint stimulation) increased the efficiency of the small-world network of healthy humans. Comparing to that, we considered the increased efficiency of SC in our study could be interpreted that patients with a short course could only build an abnormal brain circuits incompletely (close to healthy human, far from chronic migraineurs). And this incompleteness pattern would rely on the rapid transmission of information to relieve the pain sensation.

We further detected the brain regions which were significantly changed after treatment. The related regions were left corticospinal tract, left posterior limb of the internal capsule, right cingulum hippocampus, left fornix, left superior 
longitudinal fasciculus, right tapetum, pontine crossing tract and right medial lemniscus (Table 2, Figure 3). Within them, LC had four regions decreased in nodal efficiency after acupuncture. The cingulum hippocampus, which was the essential structure with the limbic system, was related to cognitive impairment and emotional change on trigeminal neuralgia and chronic pain patients [47]. The fornix had a similar function with cingulum, they both participated in affective alteration in trigeminal neuralgia [48]. The pontine crossing tract and right medial lemniscus are known as parts of the brainstem, which could be responsible areas for brainstem alterations of central sensitization. Hence, we found that all the brain regions decreased after acupuncture were relevant to central sensitization.

The changes in brain regions on SC were much complex. In the results, the left corticospinal tract, left posterior limb of the internal capsule, left superior longitudinal fasciculus and right tapetum were significantly decreased after acupuncture, while the left fornix and right medial lemniscus were increased significantly (Table 2, Figure 3). Previous studies $[49,50]$ considered that the corticospinal tract, internal capsule and superior longitudinal fasciculus were all involved with sensory-motor, affective, cognitive and pain processing functions. It could be deemed that the road of acupuncture affected patients with short courses of migraine was closer to pain management, differently with chronic migraines. Besides, the significant changes in the right tapetum displayed opposite tendency on LC and SC also strengthened our speculation about the unequal ways of acupuncture to patients with different courses of migraine.

Moreover, the relevance between the efficiency of those brain regions and the courses of migraines before the first treatment was explored (Figure 4). The 
right tapetum which affected sleep quality [51] displayed a negative correlation $(p<0.05, R=-0.538)$. The left posterior limb of the internal capsule which showed a strong relationship $(p<0.001, R=-0.734)$. They could be evidence to discriminate against the development process of migraine. Deficiently, we hadn't taken multiple interfering factors into the analysis, for instance, the background of other diseases, anxiety depression scores and concomitant medication. Besides, the slightly decreased clinical behavioral indicators (headache intensity, days and frequency) in our study showed the effect of acupuncture treatment. In summary, this research illustrated the two modulation ways of acupuncture on the efficiency of the structural brain network, which potentially contribute to reveal the mechanism of acupuncture.

The limitations of this study were as follows: 1) Sampling size limited the credibility of our conclusion. 2) Lack of various interfering factors, i.e. background of other diseases, anxiety and depression scores, and concomitant medication of subjects. Multiple regression analysis should be performed to adjust the correlation coefficient. 3) Skin irritation or psychological consolation cannot be excluded. In the beginning, this research was to stimulate the real clinical acupuncture treatment. Thus, a sham acupuncture group was not taken into consideration. All those disadvantages would be considered in the future.

\section{Conclusions}

Our study found that acupuncture had two modulation ways to affect the structural brain network of migraineurs with different courses, which could decrease the global efficiency of the brain network of chronic migraines and increase the efficiency of migraines in the early stage. The responsible brain 
regions were the pontine crossing tract, left corticospinal tract, right medial lemniscus, left posterior limb of the internal capsule, right cingulum hippocampus, left fornix, left superior longitudinal fasciculus and right tapetum. The central sensitization was the important mechanism of patients with a long course of migraines, and acupuncture could alleviate this road. Patients with a short course of migraine was unequal. The nodal efficiency of the left posterior limb of the internal capsule and right tapetum provided imaging evidences for the development process of migraines. Besides, acupuncture had insignificant effects to relieve headache intensity, days and frequency. We believed our results contributed to the understanding of mechanisms on acupuncture for migraines.

\section{Abbreviations}

MWoA: migraine without aura; EEG: electroencephalogram; TMS: transcranial magnetic stimulation; fMRI: functional magnetic resonance imaging; LC: long course group; SC: short course group; HC: healthy control group; ICHD-2: international classification of headache disease; TE: echo time; TR: repetition time; DTI: diffusion tensor imaging; FSL: FMRIB software library; DTI-TK: diffusion tensor imaging toolkit; GRETNA: graph theoretical network analysis toolbox; FA: fractional anisotropy; VAS: visual analogue scale; JHU: johns hopkins university.

\section{Acknowledgments}

Not applicable.

\section{Authors' contributions}

The manuscript was drafted by WK and LKS. The research was designed by 
$\mathrm{RY}$ and $\mathrm{ZYH}$. The manuscript examined and comments were provided by RY and ZYH. LKS and WK accounted for the data production and the analysis. The acupuncture operation and treatment were performed by $X L L, C P, J L, W Y H$, CC, WY, GHL, LRJ. All authors contributed to the publication of the manuscript in the final version.

\section{Declaration of conflicting interests}

The authors declared no potential conflicts of interest with respect for the research, authorship, and/or publication of this article.

\section{Funding}

This research was funded by the National Natural Science Foundation of China (No.2007BAI20B053), the Beijing Young Teacher of Beijing Education Committee (No.YETP0723), the Young teacher project of Beijing University of Chinese medicine (2017-JYB-JS072).

\section{Availability of data and materials}

Not applicable.

\section{Ethics approval and consent to participate}

This research was approved by the institutional review board at the Dongzhimen Hospital, Beijing University of Chinese Medicine (DZMEC-KY2017-82).

\section{Consent for publication}

Not applicable. 


\begin{tabular}{|c|c|c|c|c|c|c|c|c|c|c|c|c|}
\hline & \multicolumn{2}{|c|}{ LC, $n=10$} & \multirow[b]{2}{*}{$p$-value ${ }^{\Delta}$} & \multirow[b]{2}{*}{ t value } & \multirow[b]{2}{*}{$95 \% \mathrm{Cl}$} & \multicolumn{2}{|c|}{ SC, $n=10$} & \multirow[b]{2}{*}{$p$-value ${ }^{\Delta}$} & \multirow[b]{2}{*}{ t value } & \multirow[b]{2}{*}{$95 \% \mathrm{Cl}$} & \multirow{2}{*}{$\mathrm{HC}, \mathrm{n}=10$} & \multirow{2}{*}{$\mathrm{p}$-value $\mathrm{e}^{\Delta}$} \\
\hline & Before & After & & & & Before & After & & & & & \\
\hline $\begin{array}{l}\text { Gender } \\
\text { (women) }\end{array}$ & 10 & - & - & - & - & 8 & - & - & - & - & 8 & 0.328 \\
\hline $\begin{array}{l}\text { Age } \\
\qquad \text { (Year) }\end{array}$ & $\begin{array}{c}30.1 \\
(24-46)\end{array}$ & - & - & - & - & $\begin{array}{c}27.7 \\
(20-43)\end{array}$ & - & - & - & - & $\begin{array}{c}31.8 \\
(21-45)\end{array}$ & 0.584 \\
\hline $\begin{array}{l}\text { Course } \\
\qquad \text { (Year) }\end{array}$ & $\begin{array}{c}15.4 \\
(9-30)\end{array}$ & - & - & - & - & $\begin{array}{c}3.1 \\
(0.1-4.3)\end{array}$ & - & - & - & - & - & - \\
\hline $\begin{array}{l}\text { Headache } \\
\text { intensity }\end{array}$ & $\begin{array}{c}6.65 \\
(4-9.5)\end{array}$ & $\begin{array}{c}4.6 \\
(3-8.5)\end{array}$ & $0.005 *$ & -3.727 & $(-3.29,-0.81)$ & $\begin{array}{c}6.05 \\
(2-10)\end{array}$ & $\begin{array}{c}3.7 \\
(0-7)\end{array}$ & $0.014 *$ & -3.062 & $(-4.10,-0.61)$ & - & - \\
\hline $\begin{array}{c}\text { Headache } \\
\text { days }\end{array}$ & $\begin{array}{c}5.2 \\
(1-16)\end{array}$ & $\begin{array}{c}3.3 \\
(1-8)\end{array}$ & 0.058 & -2.172 & $(-3.88,0.08)$ & $\begin{array}{c}3.85 \\
(0.5-10)\end{array}$ & $\begin{array}{l}1.95 \\
(0-4)\end{array}$ & 0.100 & -1.836 & $(-4.24,0.44)$ & - & - \\
\hline $\begin{array}{l}\text { Headache } \\
\text { frequency }\end{array}$ & $\begin{array}{c}4.2 \\
(2-8)\end{array}$ & $\begin{array}{c}3.4 \\
(1-8)\end{array}$ & 0.121 & -1.714 & $(-1.86,0.26)$ & $\begin{array}{c}3.8 \\
(1-8)\end{array}$ & $\begin{array}{c}2.9 \\
(0-12)\end{array}$ & 0.556 & -0.612 & $(-4.23,2.43)$ & - & - \\
\hline
\end{tabular}

Table 1: The fundamental information of subjects. The p-value with notation $\triangle$ was performed by Wilcoxon test (two-tailed), and the $\triangle \triangle$ was performed by the Kruskal-Wallis test. The significant level of the p-value $(<0.05)$ was displayed in $*$, The $95 \% \mathrm{Cl}$ meant a $95 \%$ confidence interval. 


\begin{tabular}{|c|c|c|c|c|c|c|c|c|c|}
\hline \multirow{2}{*}{ Topological attribution } & \multirow{2}{*}{$\mathrm{HC}$} & \multicolumn{2}{|c|}{ LC } & \multicolumn{2}{|c|}{ LC Comparison $^{\triangle}$} & \multicolumn{2}{|c|}{ SC } & \multicolumn{2}{|c|}{ SC Comparison $^{\triangle}$} \\
\hline & & Before & After & $p$-value & t value & Before & After & $p$-value & t value \\
\hline \multicolumn{10}{|l|}{ Global network } \\
\hline Small-World Property & $\begin{array}{c}1.124 \\
(1.058- \\
1.237)\end{array}$ & $\begin{array}{c}1.115 \\
(0.897-1.294)\end{array}$ & $\begin{array}{c}1.002 \\
(0.899-1.195)\end{array}$ & 0.005 & -3.633 & $\begin{array}{c}1.086 \\
(0.932-1.573)\end{array}$ & $\begin{array}{c}1.376 \\
(1.056-1.858)\end{array}$ & 0.001 & 5.139 \\
\hline \multicolumn{10}{|l|}{ Nodal networks } \\
\hline \multicolumn{10}{|l|}{ Clustering Coefficient } \\
\hline Corticospinal Tract, Left & $\begin{array}{c}0.348 \\
(0-0.485)\end{array}$ & $\begin{array}{c}0.250 \\
(0-0.833)\end{array}$ & $\begin{array}{c}0.443 \\
(0-0.819)\end{array}$ & 0.078 & 1.986 & $\begin{array}{c}0.559 \\
(0-0.717)\end{array}$ & $\begin{array}{c}0.359 \\
(0-0.529)\end{array}$ & $0.000 * *$ & -5.148 \\
\hline $\begin{array}{l}\text { Posterior Limb of } \\
\text { Internal Capsule, Left }\end{array}$ & $\begin{array}{c}0.711 \\
(0.546-1)\end{array}$ & $\begin{array}{c}0.483 \\
(0-0.695)\end{array}$ & $\begin{array}{c}0.800 \\
(0.693-1)\end{array}$ & 0.007 & 3.460 & $\begin{array}{c}0.734 \\
(0.561-1)\end{array}$ & $\begin{array}{c}0.436 \\
(0-0.582)\end{array}$ & $0.000 * *$ & -5.868 \\
\hline $\begin{array}{l}\text { Cingulum Hippocampus, } \\
\text { Right }\end{array}$ & $\begin{array}{c}0.106 \\
(0-0.429)\end{array}$ & $\begin{array}{c}0.404 \\
(0-0.589)\end{array}$ & $\begin{array}{c}0.103 \\
(0-0.333)\end{array}$ & $0.000 * *$ & -7.389 & $\begin{array}{c}0.173 \\
(0-0.424)\end{array}$ & $\begin{array}{c}0.375 \\
(0-0.727)\end{array}$ & 0.087 & 1.914 \\
\hline Fornix, Left & $\begin{array}{c}0.289 \\
(0-1)\end{array}$ & $\begin{array}{c}0.450 \\
(0-0.833)\end{array}$ & $\begin{array}{c}0.375 \\
(0-0.643)\end{array}$ & 0.218 & -1.322 & $\begin{array}{c}0.152 \\
(0-0.484)\end{array}$ & $\begin{array}{c}0.409 \\
(0-0.575)\end{array}$ & $0.000 * *$ & 4.825 \\
\hline $\begin{array}{l}\text { Superior Longitudinal } \\
\text { Fasciculus, Left }\end{array}$ & $\begin{array}{c}0.667 \\
(0.3-0.828)\end{array}$ & $\begin{array}{c}0.668 \\
(0.467-0.865)\end{array}$ & $\begin{array}{c}0.710 \\
(0.652-0.8)\end{array}$ & 0.437 & 0.813 & $\begin{array}{c}0.618 \\
(0-0.804)\end{array}$ & $\begin{array}{c}0.474 \\
(0.385-0.563)\end{array}$ & $0.000 * *$ & -5.822 \\
\hline Tapetum, Right & $\begin{array}{c}0.122 \\
(0-0.4)\end{array}$ & $\begin{array}{c}0.132 \\
(0-0.417)\end{array}$ & $\begin{array}{l}0.648 \\
(0-1)\end{array}$ & $0.000 * *$ & 4.832 & $\begin{array}{c}0.667 \\
(0-0.9)\end{array}$ & $\begin{array}{c}0.314 \\
(0-0.526)\end{array}$ & $0.000 * *$ & -7.559 \\
\hline \multicolumn{10}{|l|}{ Nodal Local Efficiency } \\
\hline Pontine Crossing Tract & $\begin{array}{c}0.522 \\
(0-1)\end{array}$ & $\begin{array}{c}0.620 \\
(0-0.806)\end{array}$ & $\begin{array}{c}0.121 \\
(0-0.597)\end{array}$ & $0.000 * *$ & -6.562 & $\begin{array}{c}0.592 \\
(0-0.811)\end{array}$ & $\begin{array}{c}0.650 \\
(0-0.805)\end{array}$ & 0.335 & 1.019 \\
\hline Medial Lemniscus, Right & $\begin{array}{c}0.446 \\
(0-0.847)\end{array}$ & $\begin{array}{l}0.733 \\
(0-1)\end{array}$ & $\begin{array}{l}0.544 \\
(0-1)\end{array}$ & 0.216 & -1.330 & $\begin{array}{c}0.131 \\
(0-0.541)\end{array}$ & $\begin{array}{c}0.614 \\
(0-0.777) \\
\end{array}$ & $0.000 * *$ & 6.560 \\
\hline
\end{tabular}

Table 2: The analysis list of the topological attributions. The comparison with notation $\Delta$ was performed by paired Wilcoxon test, the significant level of the $p$-value $(<0.001$, two-tailed, adjusted by Bonferroni) was displayed with $* *$. 


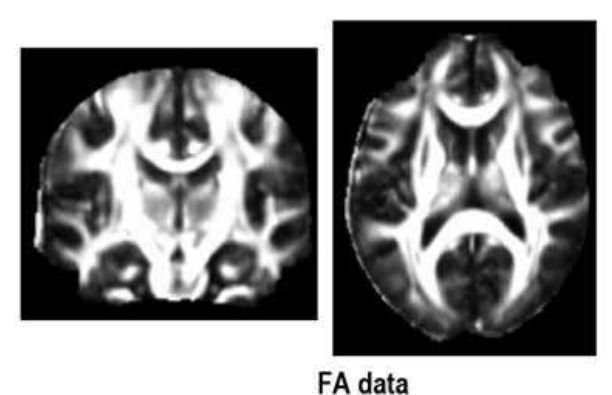

FA data

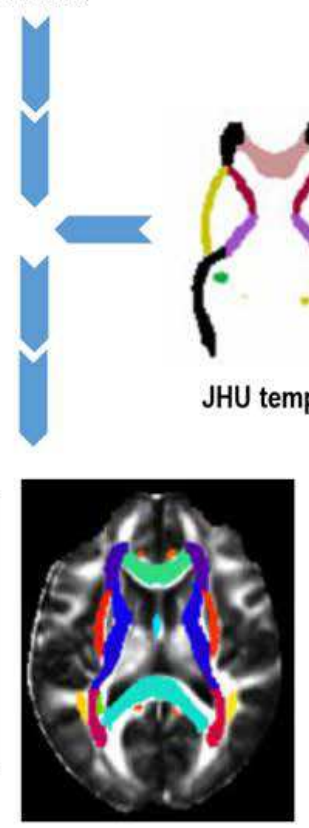

Processed data overlapped the JHU template

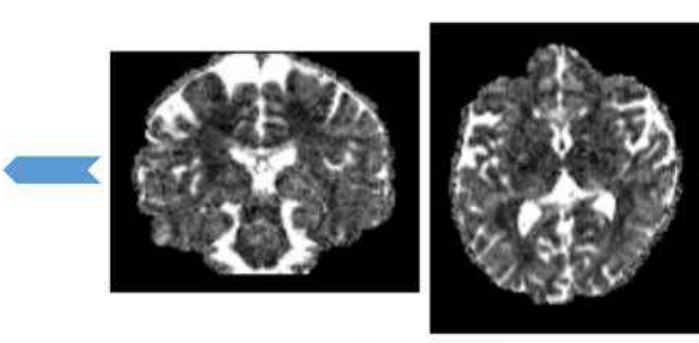

Tensor data

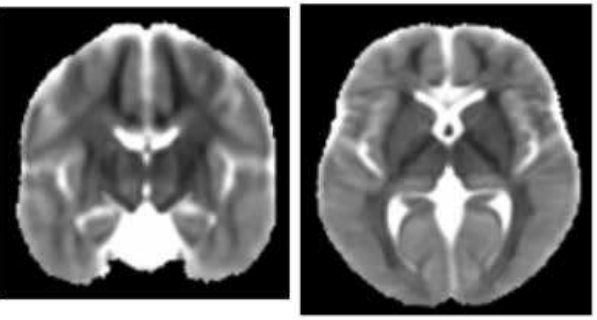

Enhanced Human Brain Atlas template

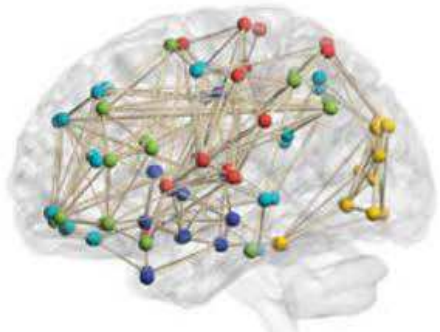

Brain network

Figure 1: Scheme of the data process. The original data was normalized by the enhanced human brain atlas template to generate the FA (fractional anisotropy) data and then overlapped the JHU (Johns Hopkins University) template to establish the structural brain network. 

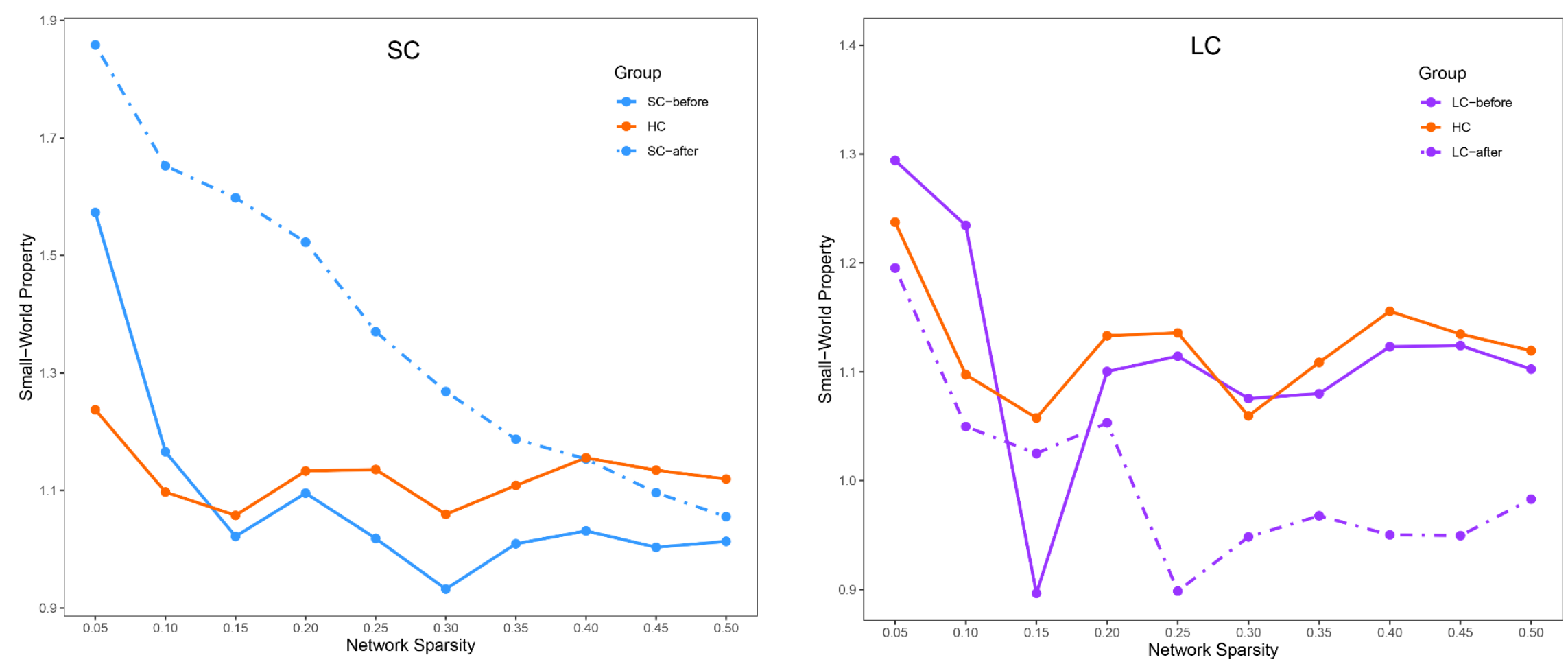

Figure 2: The changes of small-world property on LC and SC. The dotted line represented the small-world property after the final treatment. The solid line represented the small-world property before the first treatment. 


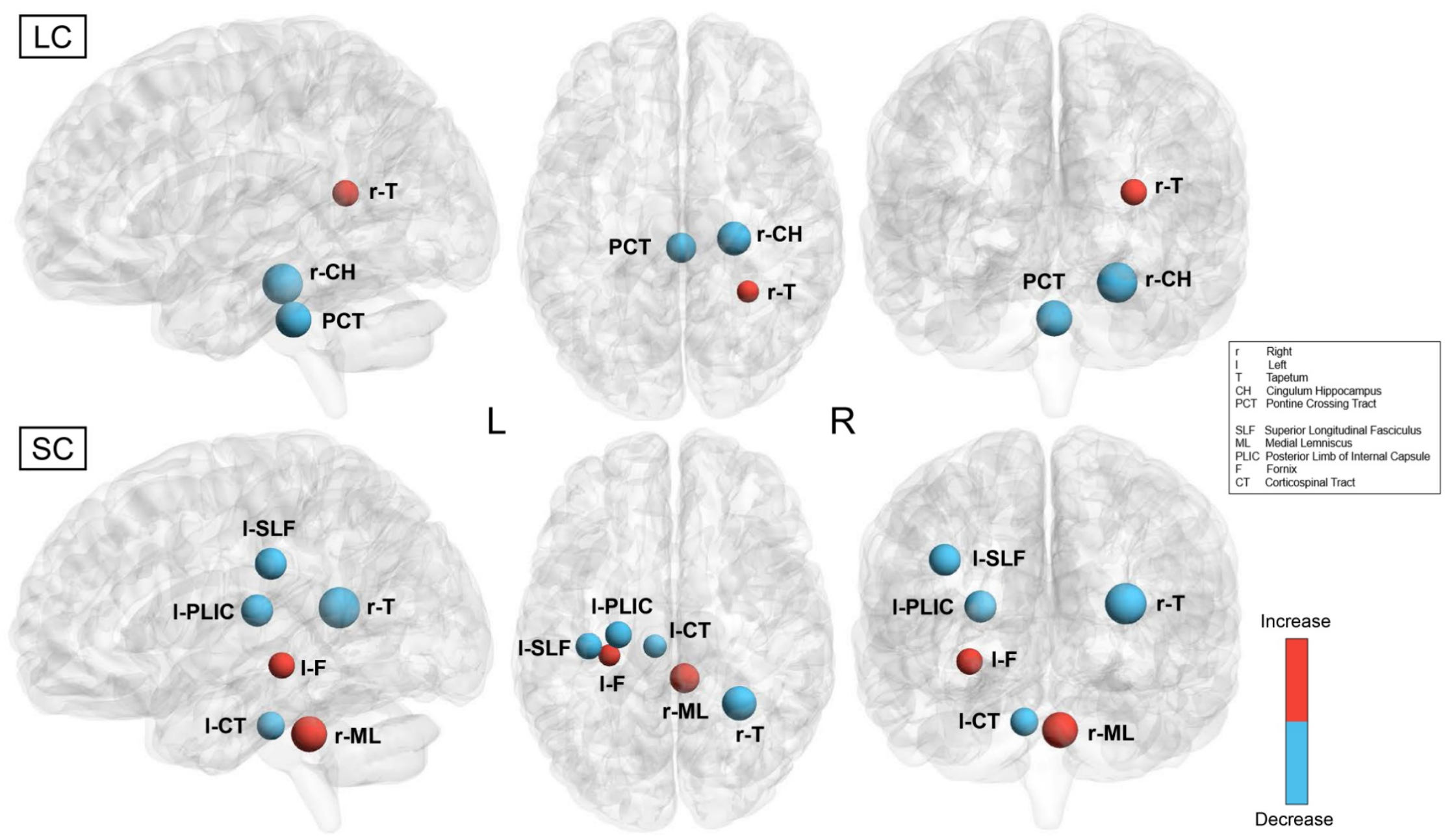

Figure 3: The brain regions which were significantly changed after treatment $(p<0.001)$. The color red represented the increase of nodal efficiency and color blue represented the decrease. The sizes of brain regions were dependent on t value in Table 2. 

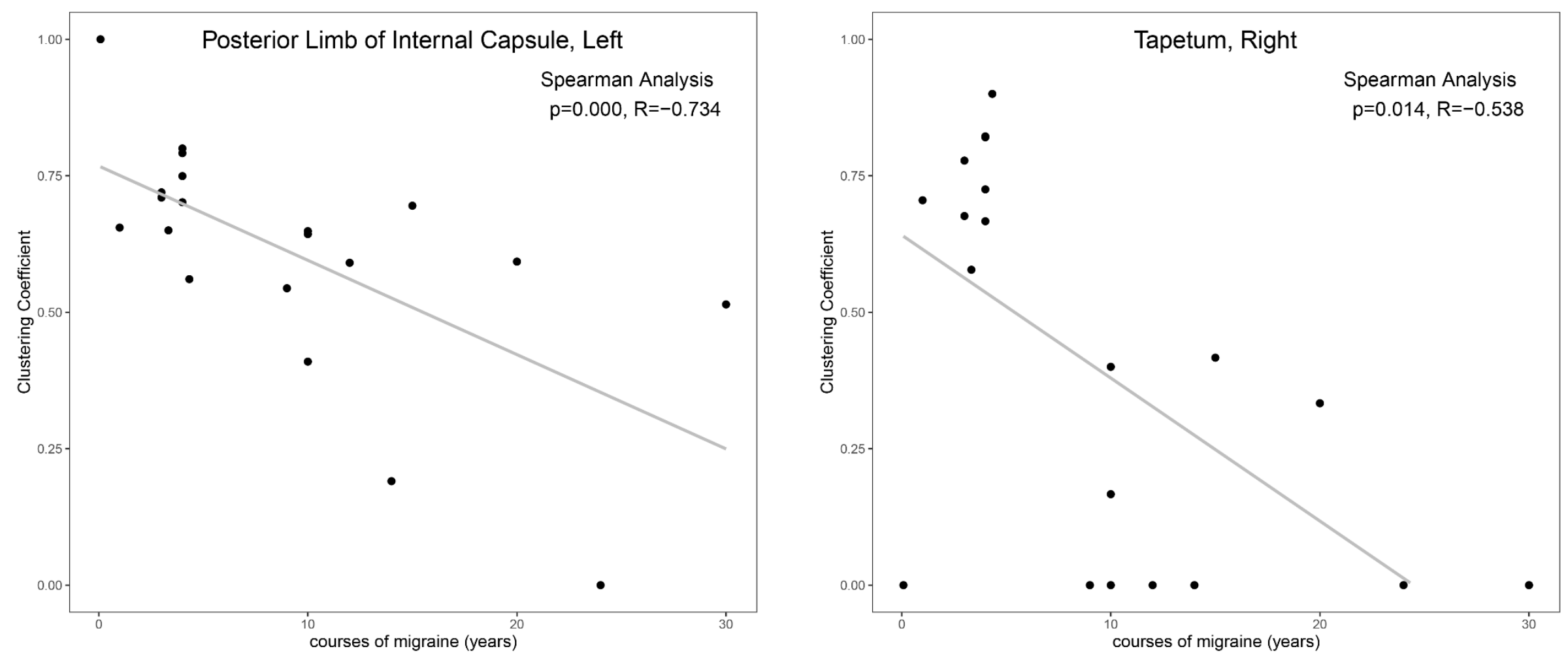

Figure 4: The brain regions which were relevant to the courses of migraine before acupuncture. The posterior limb of the internal capsule and right tapetum showed a negative correlation with the courses of migraines. 


\section{REFERENCE}

1. Lipton, R.B., et al., Migraine prevalence, disease burden, and the need for preventive therapy. Neurology, 2007. 68(5): p. 343-9.

2. Dodick, D.W., Migraine. The Lancet, 2018. 391(10127): p. 1315-1330.

3. Zhao, L., et al., The Long-term Effect of Acupuncture for Migraine Prophylaxis: A Randomized Clinical Trial. JAMA Intern Med, 2017. 177(4): p. 508-515.

4. Liu, L., et al., Acupuncture as prophylaxis for chronic migraine: a protocol for a single-blinded, double-dummy randomised controlled trial. BMJ Open, 2018. 8(5): p. e020653.

5. Zhang, N., et al., Systematic Review: Acupuncture vs Standard Pharmacological Therapy for Migraine Prevention. Headache, 2020. 60(2): p. 309-317.

6. Linde, K., et al., Acupuncture for the prevention of episodic migraine. Cochrane Database Syst Rev, 2016. 2016(6): p. Cd001218.

7. Da Silva, A.N., Acupuncture for migraine prevention. Headache, 2015. 55(3): p. 470-3.

8. Wang, L.P., et al., Efficacy of acupuncture for migraine prophylaxis: a singleblinded, double-dummy, randomized controlled trial. Pain, 2011. 152(8): p. 186471.

9. Chen, Y.Y., et al., Acupuncture versus propranolol in migraine prophylaxis: an indirect treatment comparison meta-analysis. J Neurol, 2020. 267(1): p. 14-25.

10. Yu, H., et al., Modulation of Spectral Power and Functional Connectivity in Human Brain by Acupuncture Stimulation. IEEE Trans Neural Syst Rehabil Eng, 2018. 26(5): p. 977-986.

11. Sun, Z.G., et al., Effect of acupuncture at ST36 on motor cortical excitation and inhibition. Brain Behav, 2019. 9(9): p. e01370.

12. Li, Z., et al., Acupuncture modulates the abnormal brainstem activity in migraine without aura patients. Neuroimage Clin, 2017. 15: p. 367-375.

13. van den Heuvel, M.P. and H.E. Hulshoff Pol, Exploring the brain network: a review on resting-state fMRI functional connectivity. Eur Neuropsychopharmacol, 2010. 20(8): p. 519-34.

14. Coppola, G., et al., Resting state connectivity between default mode network and insula encodes acute migraine headache. Cephalalgia, 2018. 38(5): p. 846-854.

15. Mickleborough, M.J., et al., Attentional Network Differences Between Migraineurs and Non-migraine Controls: fMRI Evidence. Brain Topogr, 2016. 29(3): p. 419-28.

16. Androulakis, X.M., et al., Modulation of salience network intranetwork resting state functional connectivity in women with chronic migraine. Cephalalgia, 2018. 38(11): p. 1731-1741.

17. Li, Z., et al., The altered right frontoparietal network functional connectivity in migraine and the modulation effect of treatment. Cephalalgia, 2017. 37(2): p. 161-176.

18. Li, K., et al., The effects of acupuncture treatment on the right frontoparietal network in migraine without aura patients. J Headache Pain, 2015. 16: p. 518.

19. Liu, J., et al., The trade-off between wiring cost and network topology in white matter structural networks in health and migraine. Exp Neurol, 2013. 248: p. 196204. 
20. Li, K., et al., Abnormal rich club organization and impaired correlation between structural and functional connectivity in migraine sufferers. Brain Imaging Behav, 2017. 11(2): p. 526-540.

21. Liu, J., et al., Integration of white matter network is associated with interindividual differences in psychologically mediated placebo response in migraine patients. Hum Brain Mapp, 2017. 38(10): p. 5250-5259.

22. Honningsvåg, L.M., et al., White matter hyperintensities and headache: A population-based imaging study (HUNT MRI). Cephalalgia, 2018. 38(13): p. 19271939.

23. Kattem Husøy, A., et al., Diffusion tensor imaging in middle-aged headache sufferers in the general population: a cross-sectional population-based imaging study in the Nord-Trøndelag health study (HUNT-MRI). J Headache Pain, 2019. 20(1): p. 78.

24. Szabó, N., et al., White matter microstructural alterations in migraine: a diffusionweighted MRI study. Pain, 2012. 153(3): p. 651-6.

25. Yu, D., et al., Axonal loss of white matter in migraine without aura: a tract-based spatial statistics study. Cephalalgia, 2013. 33(1): p. 34-42.

26. Liu, J., et al., Migraine-related gray matter and white matter changes at a 1-year follow-up evaluation. J Pain, 2013. 14(12): p. 1703-8.

27. Zhang, J., et al., Assessment of gray and white matter structural alterations in migraineurs without aura. J Headache Pain, 2017. 18(1): p. 74.

28. Uggetti, C., et al., Migraine with aura and white matter lesions: an MRI study. Neurol Sci, 2017. 38(Suppl 1): p. 11-13.

29. Neeb, L., et al., No microstructural white matter alterations in chronic and episodic migraineurs: a case-control diffusion tensor magnetic resonance imaging study. Headache, 2015. 55(2): p. 241-51.

30. Planchuelo-Gómez, Á., et al., White matter changes in chronic and episodic migraine: a diffusion tensor imaging study. J Headache Pain, 2020. 21(1): p. 1.

31. Chong, C.D. and T.J. Schwedt, Migraine affects white-matter tract integrity: A diffusion-tensor imaging study. Cephalalgia, 2015. 35(13): p. 1162-71.

32. Arkink, E.B., et al., Microstructural white matter changes preceding white matter hyperintensities in migraine. Neurology, 2019. 93(7): p. e688-e694.

33. Farahani, F.V., W. Karwowski, and N.R. Lighthall, Application of Graph Theory for Identifying Connectivity Patterns in Human Brain Networks: A Systematic Review. Front Neurosci, 2019. 13: p. 585.

34. van den Heuvel, M.P. and O. Sporns, Rich-club organization of the human connectome. J Neurosci, 2011. 31(44): p. 15775-86.

35. Sporns, O., Graph theory methods: applications in brain networks. Dialogues Clin Neurosci, 2018. 20(2): p. 111-121.

36. Olesen, J. and T.J. Steiner, The International classification of headache disorders, 2nd edn (ICDH-II). J Neurol Neurosurg Psychiatry, 2004. 75(6): p. 808-11.

37. Smith, S.M., et al., Advances in functional and structural MR image analysis and implementation as FSL. Neuroimage, 2004. 23 Suppl 1: p. S208-19.

38. Wang, Y., et al., DTI registration in atlas based fiber analysis of infantile Krabbe disease. Neuroimage, 2011. 55(4): p. 1577-86.

39. Zhang, S. and K. Arfanakis, Evaluation of standardized and study-specific diffusion 
tensor imaging templates of the adult human brain: Template characteristics, spatial normalization accuracy, and detection of small inter-group FA differences. Neuroimage, 2018. 172: p. 40-50.

40. Wang, J., et al., GRETNA: a graph theoretical network analysis toolbox for imaging connectomics. Front Hum Neurosci, 2015. 9: p. 386.

41. Rubinov, M. and O. Sporns, Complex network measures of brain connectivity: uses and interpretations. Neuroimage, 2010. 52(3): p. 1059-69.

42. Tfelt-Hansen, P.C. and P.J. Koehler, One hundred years of migraine research: major clinical and scientific observations from 1910 to 2010. Headache, 2011. 51(5): p. 752-78.

43. Ji, R.R., et al., Neuroinflammation and Central Sensitization in Chronic and Widespread Pain. Anesthesiology, 2018. 129(2): p. 343-366.

44. Aurora, S.K., A. Kulthia, and P.M. Barrodale, Mechanism of chronic migraine. Curr Pain Headache Rep, 2011. 15(1): p. 57-63.

45. Su, M. and S. Yu, Chronic migraine: A process of dysmodulation and sensitization. Mol Pain, 2018. 14: p. 1744806918767697.

46. Aurora, S.K. and M.F. Brin, Chronic Migraine: An Update on Physiology, Imaging, and the Mechanism of Action of Two Available Pharmacologic Therapies. Headache, 2017. 57(1): p. 109-125.

47. Vaculik, M.F., et al., Selective hippocampal subfield volume reductions in classic trigeminal neuralgia. Neuroimage Clin, 2019. 23: p. 101911.

48. Hayes, D.J., et al., Affective Circuitry Alterations in Patients with Trigeminal Neuralgia. Front Neuroanat, 2017. 11: p. 73.

49. Tian, T., et al., Brain white matter plasticity and functional reorganization underlying the central pathogenesis of trigeminal neuralgia. Sci Rep, 2016. 6: p. 36030.

50. Liu, P., et al., White matter microstructure alterations in primary dysmenorrhea assessed by diffusion tensor imaging. Sci Rep, 2016. 6: p. 25836.

51. Mander, B.A., et al., White Matter Structure in Older Adults Moderates the Benefit of Sleep Spindles on Motor Memory Consolidation. J Neurosci, 2017. 37(48): p. 11675-11687. 


\section{Figures}
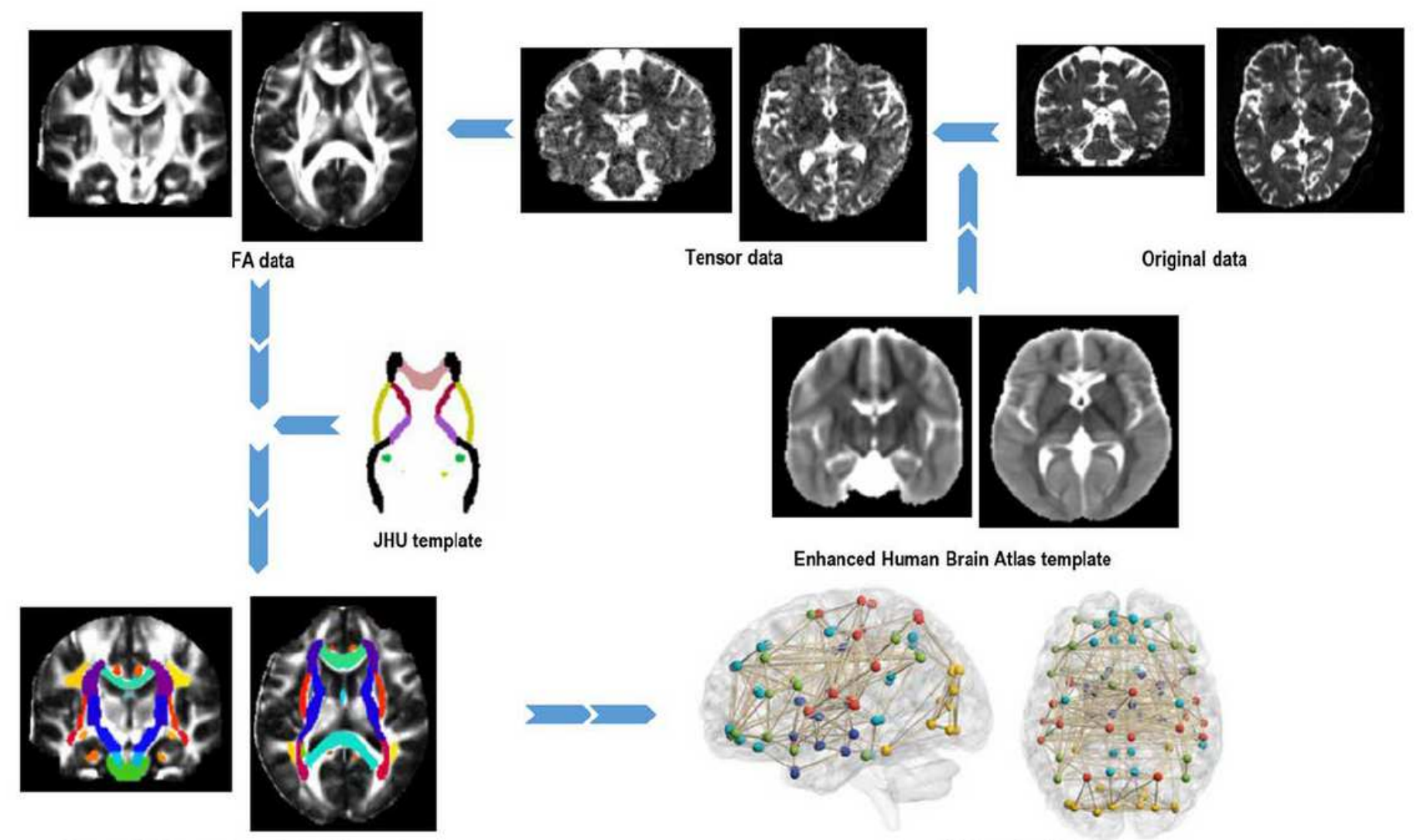

Processed data overlapped the JHU template

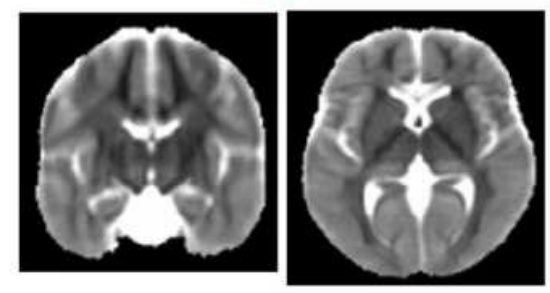

Enhanced Human Brain Atlas template

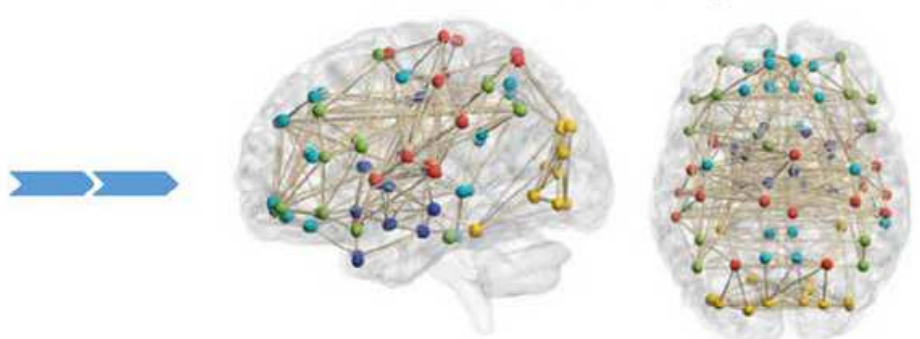

Brain network

\section{Figure 1}

Scheme of the data process. The original data was normalized by the enhanced human brain atlas template to generate the FA (fractional anisotropy) data and then overlapped the JHU (Johns Hopkins University) template to establish the structural brain network. 

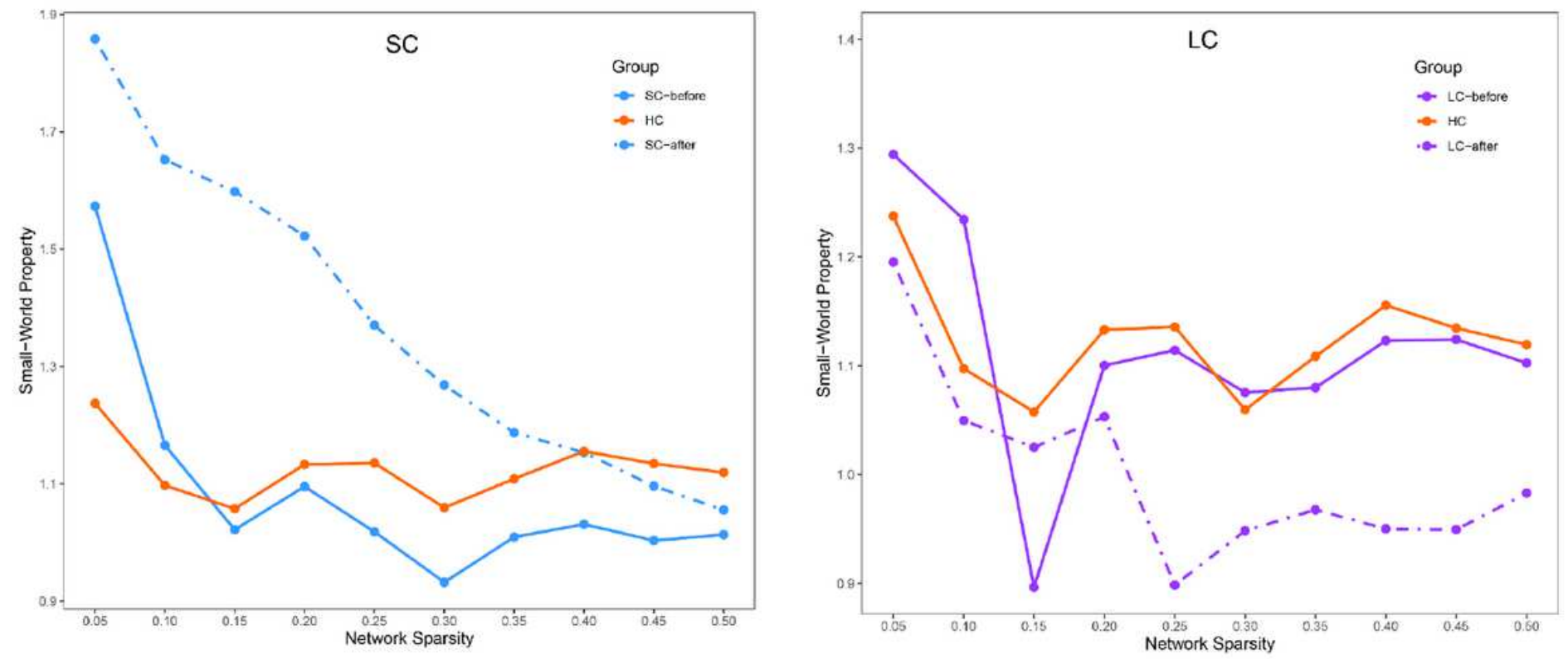

\section{Figure 2}

The changes of small-world property on LC and SC. The dotted line represented the small-world property after the final treatment. The solid line represented the small-world property before the first treatment.
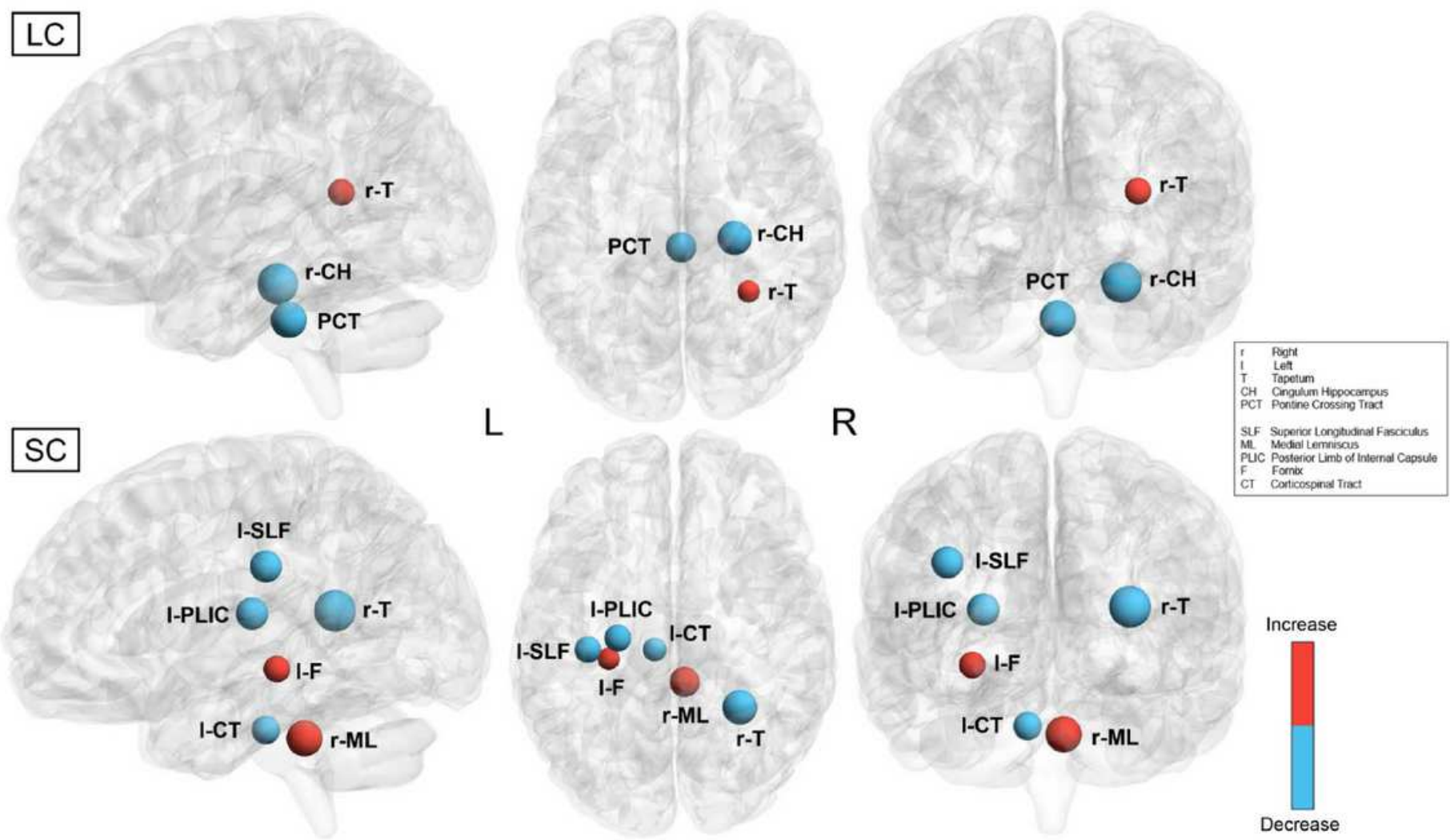

\section{Figure 3}


The brain regions which were significantly changed after treatment $(p<0.001)$. The color red represented the increase of nodal efficiency and color blue represented the decrease. The sizes of brain regions were dependent on $t$ value in Table 2.
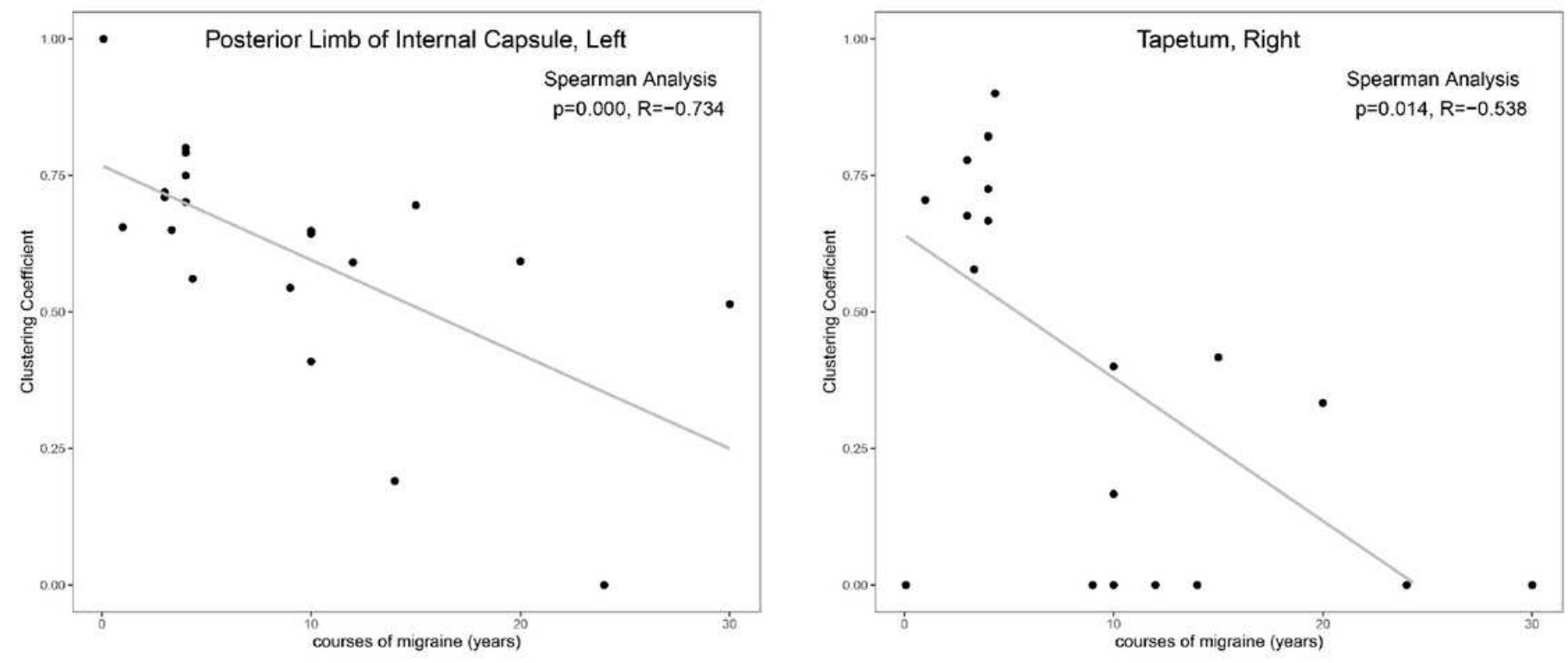

Figure 4

The brain regions which were relevant to the courses of migraine before acupuncture. The posterior limb of the internal capsule and right tapetum showed a negative correlation with the courses of migraines. 